\title{
Influence of Impurities on the Radiation Response of the TlBr Semiconductor Crystal
}

\author{
Robinson Alves dos Santos, ${ }^{1}$ Carlos Henrique de Mesquita, \\ Júlio Batista Rodrigues da Silva, ${ }^{1}$ Caue de Melo Ferraz, ${ }^{1}$ \\ Fabio Eduardo da Costa, ${ }^{1}$ João Francisco Trencher Martins, ${ }^{1}$ \\ Roseli Fernandes Gennari, ${ }^{2}$ and Margarida Mizue Hamada ${ }^{1}$ \\ ${ }^{1}$ Institute of Nuclear and Energy Research, IPEN-CNEN/SP, São Paulo, SP, Brazil \\ ${ }^{2}$ Institute of Physics, IFUSP, São Paulo, SP, Brazil \\ Correspondence should be addressed to Margarida Mizue Hamada; mmhamada@ipen.br
}

Received 1 June 2016; Revised 1 November 2016; Accepted 10 November 2016; Published 18 January 2017

Academic Editor: Pavel Lejcek

Copyright (C) 2017 Robinson Alves dos Santos et al. This is an open access article distributed under the Creative Commons Attribution License, which permits unrestricted use, distribution, and reproduction in any medium, provided the original work is properly cited.

\begin{abstract}
Two commercially available TlBr salts were used as the raw material for crystal growths to be used as radiation detectors. Previously, $\mathrm{TlBr}$ salts were purified once, twice, and three times by the repeated Bridgman method. The purification efficiency was evaluated by inductively coupled plasma mass spectroscopy (ICP-MS), after each purification process. A compartmental model was proposed to fit the impurity concentration as a function of the repetition number of the Bridgman growths, as well as determine the segregation coefficients of impurities in the crystals. The crystalline structure, the stoichiometry, and the surface morphology of the crystals were evaluated, systematically, for the crystals grown with different purification numbers. To evaluate the crystal as a radiation semiconductor detector, measurements of its resistivity and gamma-ray spectroscopy were carried out, using ${ }^{241} \mathrm{Am}$ and ${ }^{13} 3 \mathrm{Ba}$ sources. A significant improvement of the radiation response was observed in function of the crystal purity.
\end{abstract}

\section{Introduction}

The main physical semiconductor properties required for the production of room temperature semiconductor detectors are (a) high atomic number and density for high stopping power, (b) band gap large enough to maintain leakage currents low at room temperature, and (c) large mobility-lifetime products $(\mu \tau)$ for electrons and holes aiming at efficient charge collection $[1,2]$. TlBr has emerged as a particularly interesting material as room temperature semiconductor in view of its wide band gap $(2.68 \mathrm{eV})$ and its large density $\left(7.5 \mathrm{~g} / \mathrm{cm}^{3}\right)$. TlBr crystals are composed of high atomic number elements $\left(Z_{\mathrm{Tl}}=81\right.$ and $\left.Z_{\mathrm{Br}}=35\right)$ and show high resistivity $\left(>10^{10} \Omega \mathrm{cm}\right)$ [2-8]. These are important factors in applications where compact and small thickness detectors are necessary for X-and gamma-ray measurements [2].

The performance of a radiation semiconductor detector depends on several factors related to the crystal quality, such as the carrier lifetime, mobility, crystallographic imperfections, and the impurity concentrations present in the crystal. Several studies on the preparation of $\mathrm{TlBr}$ detectors have been carried out and improvements in the methodology of purification, growth, and characterization of the crystals have been described, aiming to achieve all these factors [2-7]. However, as it can be observed in the literature [1, 8-12], the $\mathrm{TlBr}$ detector limitations are not yet completely resolved: primarily, the low collection efficiency of charge carriers, a fact that is probably caused by impurities and defects created in the crystal growth or in the surface treatment process. There is a consensus in the literature that the TlBr crystal purity is a crucial factor for its optimal performance as a radiation detector [2-6].

In this work, some aspects of the crystal impurity influence on the detector performance were evaluated by systematic measurements of the gamma-ray spectrometry 
and resistivity. For purification, the crystal was grown by the Bridgman technique three times. The impurity decrease in the crystal was evaluated after each repetition number of the crystal growth, using the ICP-MS technique. The theory of compartments was used as a mathematical model to explain and to fit the data of the impurity concentration, as a function of the crystal growth repetition number.

\section{Materials and Methods}

Two commercially available TlBr salts (Merck and SigmaAldrich, in alphabetical order), with nominal purity of 99.99\%, were used as the raw salt for crystal growths. In this work, the crystals were named Salt 1 and Salt 2, but, for the sake of business ethics, the results presented here do not identify their origin. $\mathrm{TlBr}$ crystals were grown by the vertical Bridgman technique, using quartz tubes as crucibles in vacuum atmosphere. Preliminarily, the quartz tubes were submitted to a chemical treatment. The tubes were previously washed with a cleaning agent solution (Extran MA 02, Merck) and, then, filled with a solution of hydrofluoric acid (5 per cent $\mathrm{v} / \mathrm{v}$ ); after 5 minutes, the tubes were rinsed three times with demineralized water. Subsequently, the quartz tubes were submitted to a thermal treatment at $650^{\circ} \mathrm{C}$ to avoid the adhesion of the crystals on the walls of the tubes. Afterward, the $\mathrm{TlBr}$ salt was introduced into one tube, evacuated to $10^{-6}$ Torr, and sealed off. The tube with TlBr was mounted into the vertical Bridgman furnace, where the $\mathrm{TlBr}$ was melted at a temperature of $550^{\circ} \mathrm{C}$. Crystals of around $20 \mathrm{~mm}$ diameter and $60 \mathrm{~mm}$ length were obtained, with a growth rate of $1 \mathrm{~mm} / \mathrm{h}$. Following the same procedure, the crystals were grown repeatedly (three times) for purification. In this procedure, the impurities tend to migrate to the extremities of the crystal during the growth, due to the segregation of impurities along the crystal. Thus, better purity is expected to be found in the middle region. For each regrowth, the quartz tube was opened and two slice samples were taken from the crystals (Figure 1). The first sample migrated and it was taken for chemical analysis. The second sample was taken from the middle region of the ingot "middle," exactly $1.3 \mathrm{~mm}$ from the crystal middle, a region considered the prime region ( $\sim 35 \mathrm{~mm}$ thick) of the crystal, assuming that good uniformity in the impurity concentrations exists in the middle region of the ingot. Samples $(2 \times 0.65 \mathrm{~mm}$ thick slices $)$ were taken, adjacently, from the middle of the crystal, for chemical analysis and detector preparation. The "bottom" corresponds to the lower ingot extremity, which is coneshaped ( $20 \mathrm{~mm}$ thick).

A small amount of $50 \mathrm{mg}$ was taken from the "top" region and the two $0.65 \mathrm{~mm}$ samplings of the "middle" region were used to identify and determine the concentration of impurities present in each region.

The impurity concentrations of the samples, taken from slices after each growth, were measured in an ICP-MS (inductively coupled plasma mass spectrometer, mod. Elan 6100 ICP-MS, PerkinElmer, USA). Previously, samples had

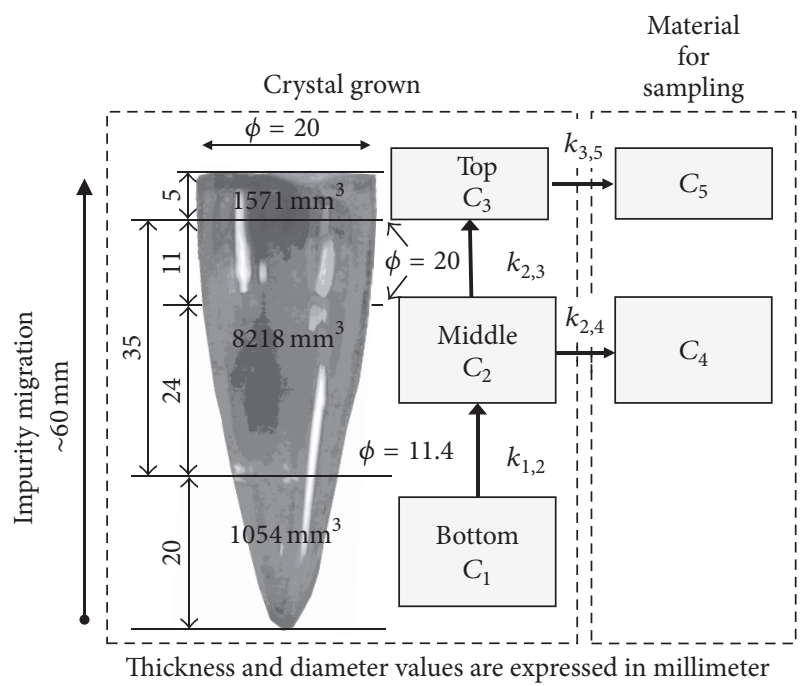

FIGURE 1: Compartmental model proposed to explain the migration of impurities in the TlBr crystal. The values of the constant $k_{i, j}$ are in Table 2.

been digested in a mixture of nitric acid (65\%, Merck) and hydrogen peroxide ( $30 \%$, Merck) by closed-vessel microwave digestion. Five impurity elements were found in the raw material: barium $(\mathrm{Ba})$, calcium $(\mathrm{Ca})$, lithium $(\mathrm{Li})$, chromium $(\mathrm{Cr})$, and copper $(\mathrm{Cu})$. The concentrations of $\mathrm{Cu}$ and $\mathrm{Cr}$ in the raw material were already in their limit of detection (0.02 ppm for $\mathrm{Cu}$ and $0.04 \mathrm{ppm}$ for $\mathrm{Cr}$ ). The sample concentrations were determined through calibration with certified single reference material. The samples were measured in 10 replicates and the results represented by the arithmetic mean and the standard deviation (mean \pm SD). The KruskalWallis One-Way Analysis of Variance on Ranks was applied to identify significant differences among the crystal impurity concentrations, compared to those found in the raw salt. The statistical calculations were performed with the SigmaStat for Windows Version 1.0 (Jandel Co. USA). The impurities were expressed in parts per million (ppm).

The theory of compartments was used as a mathematical model to explain and to fit the data of the impurity concentration as a function of the crystal growth repetition number. In the mathematical basis of a generic compartmental model, the constants $k$ will appear denoting the outputs $\left(k_{i, j}\right)$ and inputs $\left(k_{j, i}\right)$ for each compartment. In the present study, the $C_{2}$ compartment (the central region of the crystal) receives a fraction of the impurities from the lower region $\left(k_{j}=1\right.$, $i=2)$ and leaches its impurities $\left(k_{i}=2, j=3\right)$ to the top compartment $\left(C_{3}\right)$ or the upper region of the crystal. In the theory of compartmental analysis, it is assumed that the variation in the contents of the $i$ th compartment $C_{i}$ (here $C_{i}=$ impurity concentration), as a function of the variable $x$ (here $x=$ number of growth repetitions), may be equated as follows:

$$
\frac{d C_{i}(x)}{d x}=-\sum_{i=1 ; i \neq j}^{N} k_{i, j} \cdot C_{i}(x)+\sum_{j=1 ; j \neq i}^{N} k_{j, i} \cdot C_{j}(x),
$$


where $k_{i, j}$ is the constant fraction of the impurity migration from the compartment (crystal region) $i$ to compartment $j, k_{j, i}$ is the constant fraction of the impurity migration from the compartment $j$ to compartment $i$, and $N$ is the total number of compartments. Particularly, in this work, $N=5$ and $k$ constants are expressed as $x^{-1}$, that is, the inverse of Bridgman growth repetition number.

The compartmental model proposed in this work to explain the migration of impurities is shown in Figure 1. The $C_{1}$ compartment $\left(V_{C 1}=1054 \mathrm{~mm}^{3}\right)$ is assumed, physically, as being the bottom conical region which extends from zero to two millimeters in thickness $\left(\phi_{\text {minor }}=2.2 \mathrm{~mm}\right.$, $\left.\phi_{\text {major }}=11.4 \mathrm{~mm}\right)$. The $C_{2}$ compartment $\left(V_{C 2}=8218 \mathrm{~mm}^{3}\right)$ is the middle region with $35 \mathrm{~mm}$ thickness, being $24 \mathrm{~mm}$ in the conical region $\left(\phi_{\text {minor }}=11.4 \mathrm{~mm}, \phi_{\text {major }}=20 \mathrm{~mm}\right)$ and $11 \mathrm{~mm}$ in the cylindrical region $(\phi=20 \mathrm{~mm})$. The $C_{3}$ compartment $\left(1571 \mathrm{~mm}^{3}\right)$ corresponds to the top cylindrical region with $5 \mathrm{~mm}$ thickness $(\phi=20 \mathrm{~mm})$. The $C_{4}$ and $C_{5}$ compartments are located outside of the crystal region. They represent the material taken from the crystal for analysis. The accumulative $C_{4}$ compartment corresponds to the $1.3 \mathrm{~mm}$ thick slice samples, removed from $C_{2}$, at 1st, 2nd, and 3rd growth, used for chemical analysis and detector spectrometry characterization. The accumulative $C_{5}$ compartment refers to the $5 \mathrm{~mm}$ thick slice samples taken from $C_{3}$, at 1st, $2 \mathrm{nd}$, and 3 rd growth, to remove the top region where the impurities tend to migrate.
The core of this model, that is, the $C_{1}, C_{2}$, and $C_{3}$ compartments, may be defined, mathematically, as the firstorder differential equation system, shown as follows:

$$
\begin{aligned}
& \frac{d C_{1}}{d x}=-k_{1,2} \cdot C_{1} \\
& \frac{d C_{2}}{d x}=+k_{1,2} \cdot C_{1}-\left(k_{2,3}+k_{2,4}\right) \cdot C_{2} \\
& \frac{d C_{3}}{d x}=+k_{3,5} \cdot C_{3},
\end{aligned}
$$

where $C_{1,0}=C_{2,0}=C_{3,0}$ is the impurity concentration in raw material (Table 2) and $C_{4,0}=C_{5,0}=0$.

Rewriting the equation system (2) in the matrix notation and assuming the algebraic feature $\sum_{i=1 ; i \neq j}^{N} k_{i, j}=k_{i, i}$, with the intent of achieving uniformity in the indexes of the array elements, we have

$$
\left[\begin{array}{ccc}
-k_{1,1} & 0 & 0 \\
k_{1,2} & -k_{2,2} & 0 \\
0 & k_{2,3} & -k_{3,3}
\end{array}\right] \cdot\left[\begin{array}{c}
C_{1} \\
C_{2} \\
C_{3}
\end{array}\right]=\left[\begin{array}{c}
\frac{d C_{1}}{d x} \\
\frac{d C_{2}}{d x} \\
\frac{d C_{3}}{d x}
\end{array}\right]
$$

By applying the Laplace transform [13] in (3) and inverting the $[k]$ matrix,

$$
\left[\begin{array}{l}
\overline{C_{1}}(s) \\
\overline{C_{2}}(s) \\
\overline{C_{3}}(s)
\end{array}\right]=\frac{1}{\Delta} \cdot\left[\begin{array}{ccc}
\left(s+k_{2,2}\right) \cdot\left(s+k_{3,3}\right) & 0 & 0 \\
k_{1,2} \cdot\left(s+k_{3,3}\right) & \left(s+k_{1,1}\right) \cdot\left(s+k_{3,3}\right) & 0 \\
k_{1,2} \cdot k_{2,3} & k_{2,3} \cdot\left(s+k_{1,1}\right) & \left(s+k_{1,1}\right) \cdot\left(s+k_{2,2}\right)
\end{array}\right] \cdot\left[\begin{array}{c}
C_{1,0} \\
C_{2,0} \\
C_{3,0}
\end{array}\right],
$$

where $\overline{C_{i}}(s)=\mathscr{L}\left(C_{i}(x)\right)$ is the Laplace transformation of $C_{i}(x)$ by changing the " $x$ variable" to one in "s-space" and $\Delta=\left(s+k_{1,1}\right) \cdot\left(s+k_{2,2}\right) \cdot\left(s+k_{3,3}\right)$. Hence,

$$
\left[\begin{array}{l}
\overline{C_{1}}(s) \\
\overline{C_{2}}(s) \\
\overline{C_{3}}(s)
\end{array}\right]=\left[\begin{array}{c}
\frac{\left(s+k_{2,2}\right) \cdot\left(s+k_{3,3}\right)}{\left(s+k_{1,1}\right) \cdot\left(s+k_{2,2}\right) \cdot\left(s+k_{3,3}\right)} \cdot C_{1,0} \\
\frac{k_{1,2} \cdot\left(s+k_{3,3}\right) \cdot C_{1,0}+\left(s+k_{1,1}\right) \cdot\left(s+k_{3,3}\right) \cdot C_{2,0}}{\left(s+k_{11}\right) \cdot\left(s+k_{2,2}\right) \cdot\left(s+k_{3,3}\right)} \\
\frac{k_{1,2} \cdot k_{2,3} \cdot C_{1,0}+k_{2,3} \cdot\left(s+k_{1,1}\right) \cdot C_{2,0}+\left(s+k_{1,1}\right) \cdot\left(s+k_{2,2}\right) \cdot C_{3,0}}{\left(s+k_{11}\right) \cdot\left(s+k_{2,2}\right) \cdot\left(s+k_{3,3}\right)}
\end{array}\right] .
$$

Finally, applying the inverse of Laplace transformation, $C_{i}(x)=\mathscr{L}^{-1}\left(C_{i}(s)=P_{i}(s) / Q(s)\right)$, using the Heaviside algorithm, where $Q(s)=\Delta$ and $P_{i}(s)$ is the numerator elements of the matrix product (6), then we have

$$
C_{1}(x)=C_{1,0} \cdot e^{-k_{1,1} \cdot x}
$$

$$
\begin{aligned}
& C_{2}(x) \\
& =\frac{k_{1,2} \cdot C_{1,0}}{k_{2,2}-k_{1,1}} \cdot e^{-k_{1,1} \cdot x}+\left(\frac{k_{1,2} \cdot C_{1,0}}{k_{1,1}-k_{2,2}}+C_{2,0}\right) \cdot e^{-k_{2,2} \cdot x}
\end{aligned}
$$




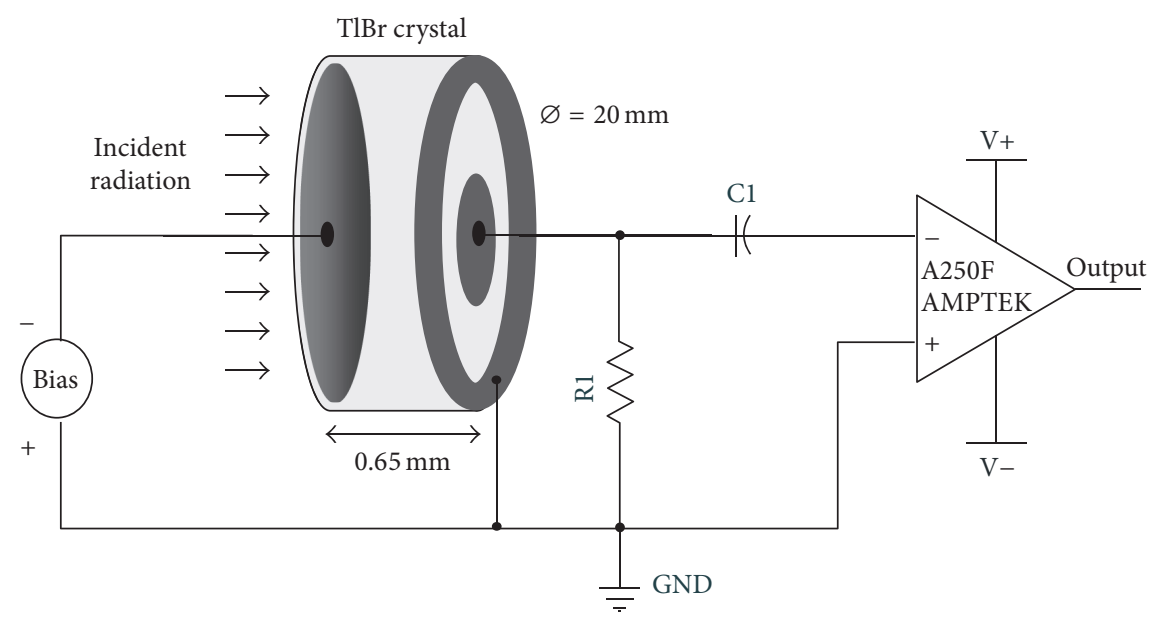

FIGURE 2: TlBr detector and preamplifier connections.

$$
\begin{aligned}
C_{3}(x) & \frac{k_{1,2} \cdot k_{2,3} \cdot C_{1,0}}{\left(k_{2,2}-k_{1,1}\right) \cdot\left(k_{3,3}-k_{1,1}\right)} \cdot e^{-k_{1,1} \cdot x} \\
& +\left(\frac{k_{1,2} \cdot k_{2,3} \cdot C_{2,0}}{\left(k_{1,1}-k_{2,2}\right) \cdot\left(k_{3,3}-k_{2,2}\right)}+\frac{k_{2,3} \cdot C_{2,0}}{k_{3,3}-k_{2,2}}\right) \\
& \cdot e^{-k_{2,2} \cdot x} \\
& +\left(\frac{k_{1,2} \cdot k_{2,3} \cdot C_{3,0}}{\left(k_{1,1}-k_{3,3}\right) \cdot\left(k_{2,2}-k_{3,3}\right)}+\frac{k_{2,3} \cdot C_{2,0}}{k_{2,2}-k_{3,3}}+C_{3,0}\right) \\
& \cdot e^{-k_{3,3} \cdot x} .
\end{aligned}
$$

The two end line compartments $C_{4}$ and $C_{5}$ have their cumulative impurities determined as follows:

$$
\begin{aligned}
& C_{4}(x)=k_{2,4} \cdot \int_{x=0}^{x} C_{2}(x) d x \\
& C_{5}(x)=k_{3,5} \cdot \int_{x=0}^{x} C_{3}(x) d x .
\end{aligned}
$$

In summary, $C_{i}(x)$ is the experimental concentration of impurities in the crystal region $i$ after the $x$ th repetition of crystal growth, $C_{i, 0}$ is the initial condition measured, experimentally, in raw material, and $k_{i, j}$ is the constant migration of impurities, determined by the nonlinear least-squares method. In this study, the compartmental calculations were made with Anacomp software [14-16].

The crystalline quality of the TlBr crystal was analyzed by X-ray diffraction (XRD). X-ray diffraction patterns were obtained in a Siemens (D5005) diffractometer with $\mathrm{CuK} \alpha$ radiation ( $2 \theta$ ranging from $20^{\circ}$ to $60^{\circ}$ ). The two sample slices from the middle crystal were prepared as a detector according to procedures described previously $[7,17,18]$. The crystal was sliced in wafers, cut transversally to direction (110), using a diamond saw, and lubricated with glycerine during the process. Crystals were cut slowly to have less damage and smaller depths in the resulting layers. Polishing, cleaning, and electrode painting were carried out, subsequently, without pause to avoid humidity deposition. The electrodes were made with colloidal carbon painting, Viatronix $^{\mathrm{TM}}$. The final dimensions of the crystal wafers were, approximately, $20 \mathrm{~mm}$ diameter and $0.65 \mathrm{~mm}$ thickness. The detectors were made with a central electrode (anode), plus a ring electrode surrounding the anode electrode. The anode electrode diameter is about $3 \mathrm{~mm}$ and the ring electrode is around $4 \mathrm{~mm}$ internal diameter plus, approximately, $10 \mathrm{~mm}$ external diameter. The area for each electrode was defined from the painting mask electrode area and the thickness, with a micrometer. Figure 2 shows a schematic diagram of the detector and its connection to the preamplifier. The output from A250F charge sensitive preamplifier was connected to a 450 EG\&G Ortec Research Amplifier at $10 \mu$ s shaping time and to EG\&G 918A Multichannel Analyzer, to obtain the pulse height spectra. The detector signal is from an electron collector. TlBr crystal detectors were excited under a $59 \mathrm{keV}$ ${ }^{241} \mathrm{Am}$ gamma source, biased with $400 \mathrm{~V}$. For resistivity measurements, the ring electrodes were disconnected and the bias current was measured with a 619 Keithley Multimeter. All measurements were carried out at room temperature, $24 \pm 2^{\circ} \mathrm{C}$.

\section{Results and Discussion}

The X-ray diffraction pattern of TlBr salt exhibited a complete set of reflections (Figure 3(a)), while the typical X-ray diffraction pattern of TlBr crystals grown in this work presented only a reflection line (Figures 3(b) and 3(c)). The diffractogram indicates that the crystal is preferentially oriented in the (110) direction (Figures 3(b) and 3(c)). It is worthwhile to observe that there was no other crystalline phase in the grown crystal since all detected peaks corresponded to the $\mathrm{TlBr}$ peaks oriented in the (110) direction. These results are in agreement with the literature $[4,10]$.

In the classical approach, to determine the calculation of the segregation coefficient $k$, some idealized hypotheses are assumed [19]: (i) the concentration of the impurity in the raw material is constant in all the extension of its distribution in the crucible; (ii) the ingot cross section is 


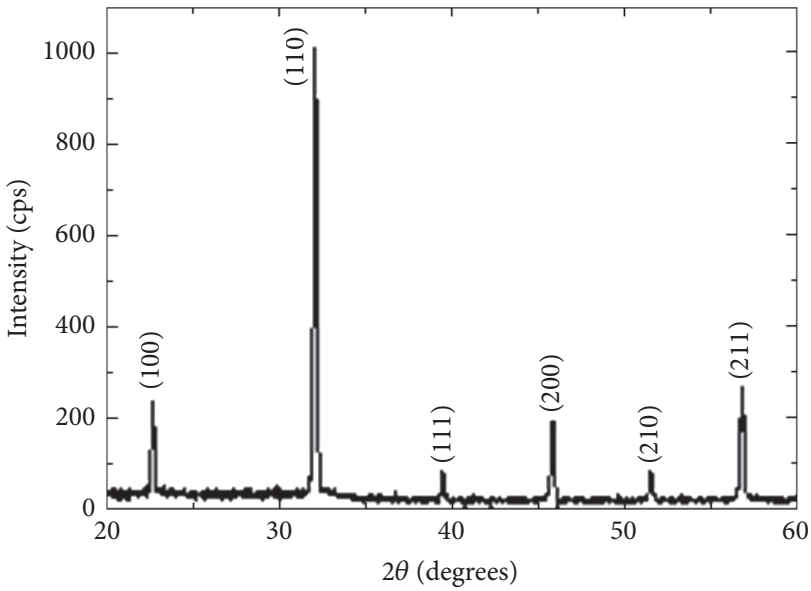

(a)

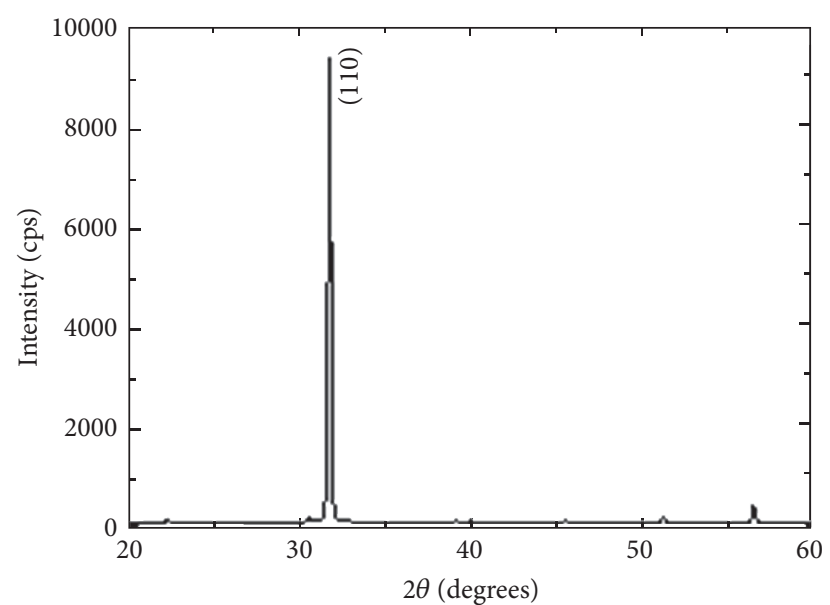

(b)

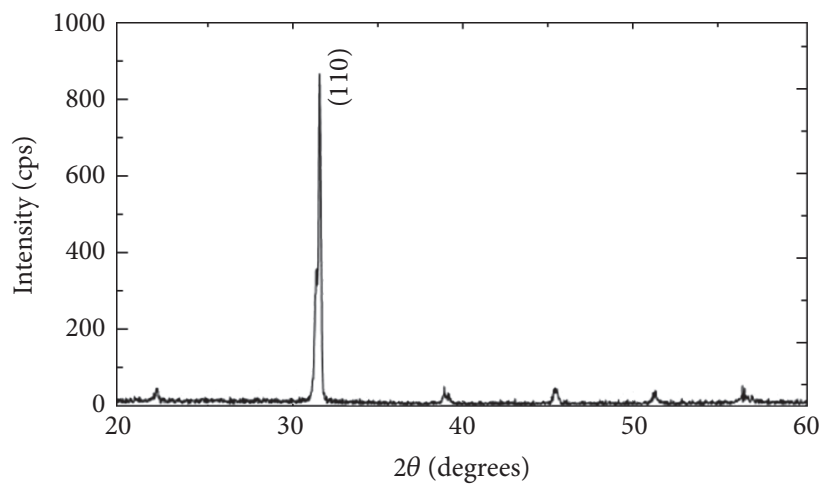

(c)

Figure 3: X-ray diffraction of $\mathrm{TlBr}$ powder (a) and $\mathrm{TlBr}$ crystal (b).

constant; (iii) the segregation coefficient is constant along the ingot length; (iv) the initial concentration in each $i$ region of the ingot corresponds to the sum of the entire ingot, divided by the number of region sections; (v) the ingot length should be greater than the melting zone length, in order to drag impurities based on solubility differences of the solidliquid phase. If all of these conditions are met, then the predictable mathematical model described in (11) may be used to calculate the concentration $C_{i}$, after zone refining:

$$
C_{i}=C_{0} \times\left[1-(1-k) \times e^{-k \cdot(i / l)}\right],
$$

where $C_{i}$ is the concentration of the impurity in the $i$ th position along the ingot; $C_{0}$ is its initial concentration; $k$ is the segregation coefficient; and $l$ is the melting zone length. Due to the experimental particularities of the Bridgman purification used in this work, the following requirements mentioned previously are not established: (1st) in the crystal growth by the Bridgman technique, the raw material needs to be fully melted previously, which is in divergence with the hypothesis number (v); (2nd) in order to promote the nucleation, the growth crucible should be, preferably, coneshaped, disregarding the hypothesis number (ii); (3th) due to the methodology applied, at each full melting, a fraction of the impurities, located in the top region, can recirculate by the Brownian movement, thereby reducing the efficiency of purification. To avoid this effect and to improve the quality of the purification process, the crucible of growth is opened and the upper portion of the crystal is cut and removed. Besides, to evaluate the effect of the impurities on the crystal performance as a radiation detector, samples were taken from the middle of the crystal, considered the prime region. Thus, due to these restrictions, the mathematical model based on zone refining, described by (11), is not suitable.

The basic assumptions applied to the formulation of the model described in Figure 1 and (6)-(10) were as follows: (i) the segregation coefficient $k<1$ (i.e., the measured impurities are more soluble in the molten fraction of the crystal); (ii) initially, the impurity concentrations in the three regions (compartments), 1 to 3 , are equal to the salt used as raw material and the initial concentrations in compartments 4 and 5 are both equal to zero; (iii) the migration coefficient $k_{i, j}$ is constant, independent of the crystal growth repetition number $x$ (i.e., the quantity of impurities that migrate from region $i$ to region $j$ is proportional to their concentration in region $i$ ); and, finally, (iv) $k_{1,2}=k_{2,3}$. This algebraic feature allows a reduction in the number of variables to be determined by the nonlinear least-squares method [20]; 
TABLE 1: Three impurity concentrations (ppm) in the TlBr salt and in the top and middle regions of the TlBr crystal, by ICP-MS. The values represent the mean one standard deviation $(N=10$ samples $)$.

\begin{tabular}{|c|c|c|c|c|c|c|}
\hline \multirow{2}{*}{\multicolumn{2}{|c|}{$\mathrm{TlBr}$ origin }} & & \multirow{2}{*}{ Crystal region } & \multicolumn{3}{|c|}{ Impurities elements (ppm) } \\
\hline & & & & $\mathrm{Ba}$ & $\mathrm{Ca}$ & $\mathrm{Li}$ \\
\hline \multirow{7}{*}{ Salt 1} & \multirow{7}{*}{ Bridgman growth step } & \multirow{3}{*}{ First } & Raw material & $5.46 \pm 0.10$ & $8.99 \pm 0.12$ & $2.39 \pm 0.10$ \\
\hline & & & Top & $5.36 \pm 0.12$ & $8.58 \pm 0.13$ & $2.34 \pm 0.14$ \\
\hline & & & Middle & $4.93^{*} \pm 0.12$ & $8.28^{*} \pm 0.07$ & $2.05 \pm 0.12$ \\
\hline & & \multirow{2}{*}{ Second } & Top & $6.38^{*} \pm 0.13$ & $8.49 \pm 0.10$ & $3.00^{*} \pm 0.12$ \\
\hline & & & Middle & $6.09^{*} \pm 0.11$ & $8.16^{*} \pm 0.13$ & $3.04^{*} \pm 0.11$ \\
\hline & & \multirow{2}{*}{ Third } & Top & $5.49 \pm 0.11$ & $8.13^{*} \pm 0.09$ & $2.76 \pm 0.11$ \\
\hline & & & Middle & $3.05^{*} \pm 0.15$ & $6.88^{*} \pm 0.12$ & $1.88^{*} \pm 0.12$ \\
\hline \multirow{7}{*}{ Salt 2} & \multirow{7}{*}{ Bridgman growth step } & \multirow{3}{*}{ First } & Raw material & $9.64 \pm 0.11$ & $7.69 \pm 0.09$ & $1.92 \pm 0.10$ \\
\hline & & & Top & $9.25^{*} \pm 0.09$ & $8.03^{*} \pm 0.09$ & $1.61^{*} \pm 0.12$ \\
\hline & & & Middle & $9.10^{*} \pm 0.11$ & $7.63^{*} \pm 0.10$ & $1.60^{*} \pm 0.07$ \\
\hline & & \multirow{2}{*}{ Second } & Top & $9.11^{*} \pm 0.12$ & $7.62 \pm 0.12$ & $1.54^{*} \pm 0.11$ \\
\hline & & & Middle & $8.96^{*} \pm 0.10$ & $7.49 \pm 0.012$ & $1.40^{*} \pm 0.06$ \\
\hline & & \multirow{2}{*}{ Third } & Top & $9.02^{*} \pm 0.09$ & $7.45^{*} \pm 0.11$ & $1.57^{*} \pm 0.09$ \\
\hline & & & Middle & $8.68^{*} \pm 0.08$ & $6.96^{*} \pm 0.12$ & $1.33^{*} \pm 0.12$ \\
\hline
\end{tabular}

${ }^{*}$ There is a statistical difference between sample value and the initial concentration of raw salt $(p<0.05)$.

TABLE 2: Impurity migration coefficients.

\begin{tabular}{lcrr}
\hline Impurities & Transfer coefficient & TlBr Powder 1 $\left(x^{-1}\right)^{\mathrm{a}}$ & TlBr Powder 2 $\left(x^{-1}\right)^{\mathrm{a}}$ \\
\hline \multirow{3}{*}{ Barium } & $k 1,2=k 2,3$ & $0.35 \pm 0.01^{\mathrm{b}}$ & $0.057 \pm 0.003^{\mathrm{b}}$ \\
& $k 2,4$ & $0.074 \pm 0.013^{\mathrm{b}}$ & $0.037 \pm 0.003^{\mathrm{b}}$ \\
\hline \multirow{3}{*}{ Calcium } & $k 3,5$ & $0.26 \pm 0.01^{\mathrm{b}}$ & $0.083 \pm 0.003^{\mathrm{b}}$ \\
& $k 1,2=k 2,3$ & $0.29 \pm 0.09^{\mathrm{b}}$ & $0.17 \pm 0.02^{\mathrm{b}}$ \\
& $k 2,4$ & $0.0076 \pm 0.0463^{\mathrm{b}}$ & $0.00070 \pm 0.00887^{\mathrm{b}}$ \\
Lithium & $k 3,5$ & $0.311 \pm 0.086^{\mathrm{b}}$ & $0.18 \pm 0.02^{\mathrm{b}}$ \\
& $k 1,2=k 2,3$ & $0.21 \pm 0.51^{\mathrm{b}}$ & $0.056 \pm 0.356^{\mathrm{b}}$ \\
\hline Mean \pm SD & $k 2,4$ & $0.004 \pm 0.200^{\mathrm{b}}$ & $0.14 \pm 0.02^{\mathrm{b}}$ \\
\hline
\end{tabular}

${ }^{\mathrm{a}} x$ : number of repeated Bridgman growths.

${ }^{\mathrm{b}}$ Regression asymptotic error (the calculated error in the last iteration).

moreover, this hypothesis is in agreement with the same rule (hypothesis number (iii)), used in the formulation of the zone refined model ( $k$ without index in (11)). The constants $k_{2,4}$ and $k_{3,5}$ depend on the size of the sliced material removed from the crystal and were numerically estimated by the fitting regression process.

The concentration of three ions ( $\mathrm{Ca}, \mathrm{Ba}$, and $\mathrm{Li}$ ) found in the crystal grown three times, sequentially, for the two salts, is presented in Table 1 and Figure 4. Comparisons among initial impurities from the two salts (raw materials) suggest that Salt 1 has less $\mathrm{Ba}(5.46 \pm 0.10 \mathrm{ppm})$ than Salt $2(9.64 \pm$ $0.11 \mathrm{ppm}$ ), while both salts have similar concentration of $\mathrm{Ca}$ $(8.99 \pm 0.12 \mathrm{ppm}$ for Salt 1 versus $7.69 \pm 0.09 \mathrm{ppm}$ for Salt 2$)$ and $\mathrm{Li}(2.39 \pm 0.10 \mathrm{ppm}$ for Salt 1 versus $1.92 \pm 0.10 \mathrm{ppm}$ for Salt 2).

For Salt 1, after the first purification, the amount of impurities in the top region was not, significantly, different from the raw salt. However, for Salt 2, major differences were found for all three impurity elements in the crystal top region. In the case of the crystal middle region, a significant difference in the crystal impurity concentrations was observed for almost all impurities, compared to those found in the raw material (Salts 1 and 2).

According to Table 1, for Salt 1, the reduction level of the impurities in the crystal middle region, after the third growth, was of $44 \%(1-(3.05 / 5.46))$ for $\mathrm{Ba}, 23 \%(1-(6.88 / 8.99))$ for $\mathrm{Ca}$, and $21 \%(1-(1.88 / 2.39))$ for $\mathrm{Li}$. On the other hand, for the second salt, the results were worse, with $10 \%$ (1 $(8.68 / 9.64))$ for $\mathrm{Ba}, 9 \%(1-(6.96 / 7.69))$ for $\mathrm{Ca}$, and $31 \%(1$ - (1.33/1.92)) for Li. Comparing the averages of $k_{1,2}=k_{2,3}$ (Table 2) for the two salts, similar results can be reached. It should be emphasized that, in the compartmental theory, there is not a rigorous commitment that $k$ parameter should be equal among the regions. However, for the same raw salt, 

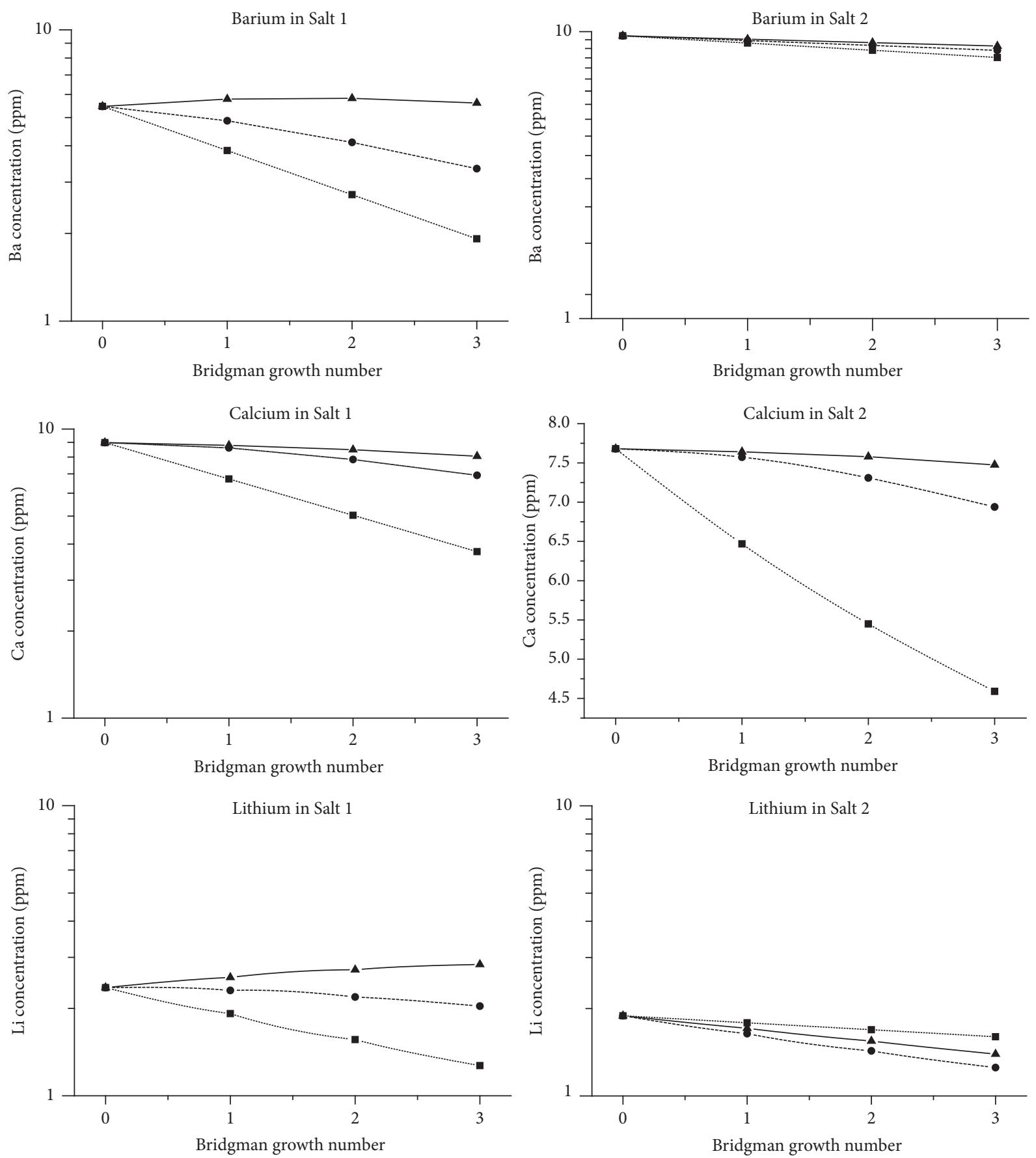

Figure 4: Concentration of impurity ions in different regions of crystals. $\mathbf{a}$ bottom region: compartment $C_{1}$; middle region: compartment $C_{2}$; $\mathbf{\Delta}$ top region: compartment $C_{3}$. Values were calculated theoretically from model described in Figure 1 and (7), (8), and (9).

the $k_{1,2}=k_{2,3}$ values found were close to each other, and thus this assumption may be made. The difference in the mean values of these two groups $(0.28 \pm 0.07$ versus $0.094 \pm 0.07)$ is greater than what could be expected by chance; hence, there is a statistically significant difference between Salts 1 and 2 ( $p=$ 0.0271). These results suggest that some unknown factors, present in Salt 2, slow down the separation of impurities along the crystal and, consequently, the choice of the commercial raw salt should be made experimentally, independent of its nominal declaration of purity.
The model shown in Figure 1 is valuable for quality control purposes. In such case, the $k_{i, j}$ parameters, which are associated with the impurity migration efficiency, may be an important auxiliary tool to design, optimize, and explain the results and processes involved in the purification of raw salt used to grow crystals. For example, to understand the rise in the concentration of impurities in the crystal middle region, increased in the second Bridgman growth (Figures 4 and 5), despite a seeming contradiction, this occurrence can be predicted and quantified by the compartmental analysis. 


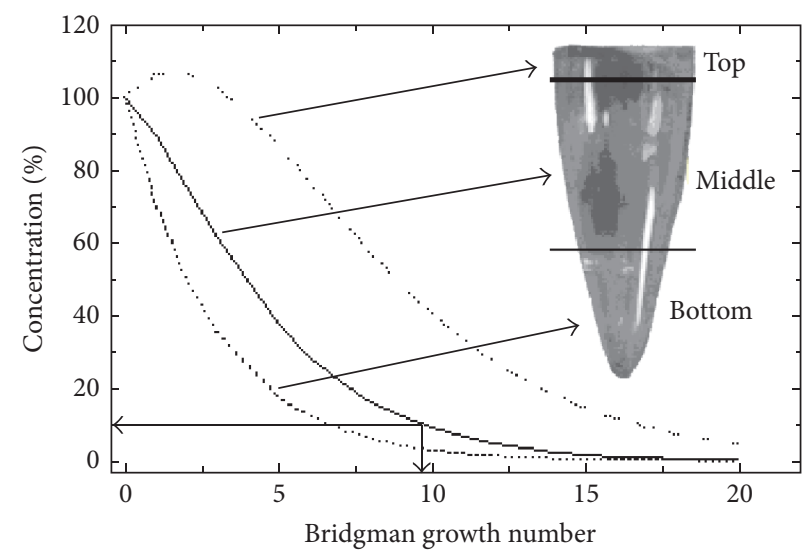

Figure 5: Predictive concentration (\%) for Salt 1 as a function of repetition number of Bridgman growth, curves calculated from the model of Figure 1 and (7), (8), and (9). To achieve 10\% of initial concentration in the crystal middle region, approximately 10 steps are required.
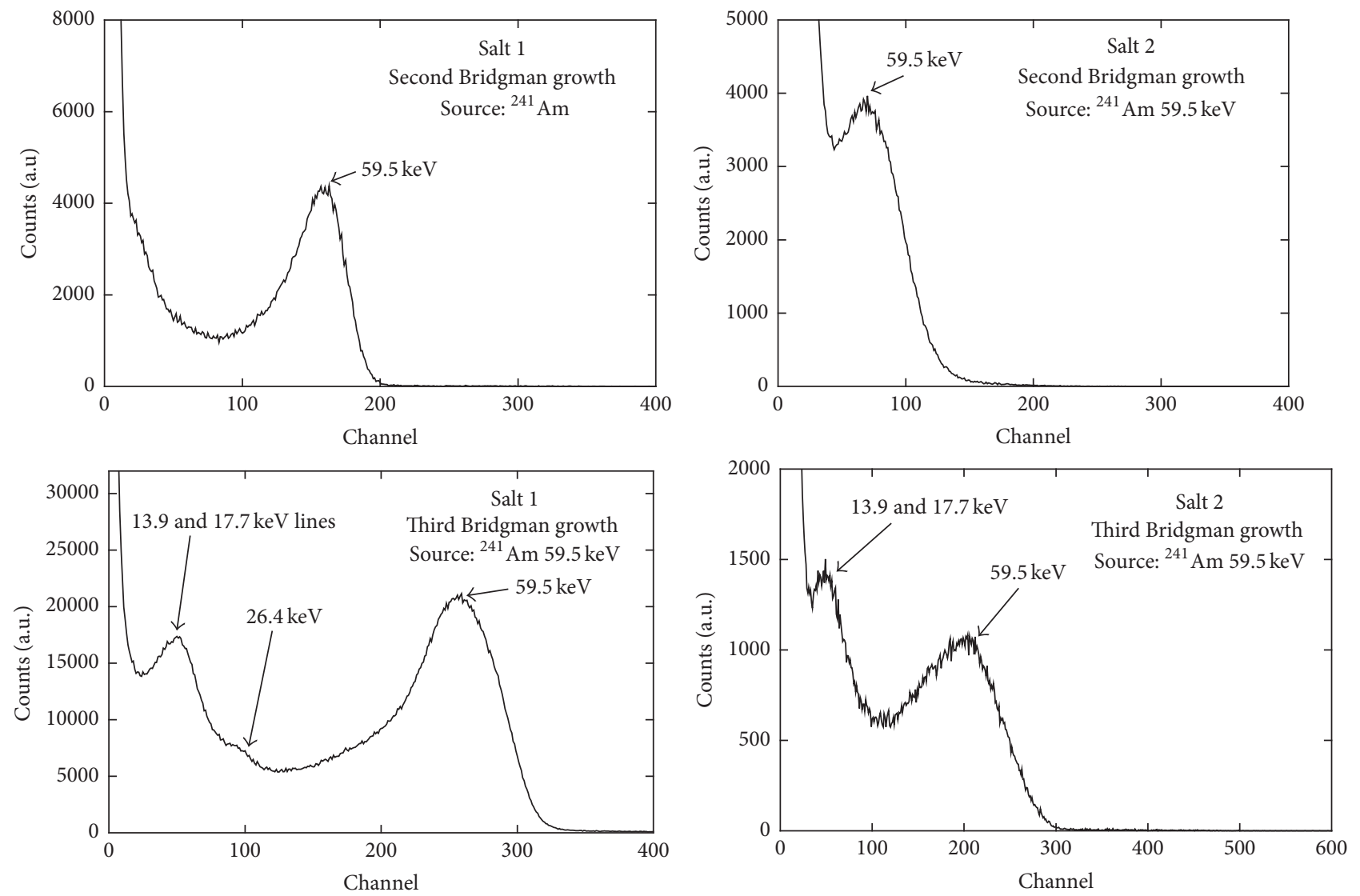

Figure 6: TlBr detector energy spectra under ${ }^{241}$ Am excitations. Detectors were prepared using TlBr samples from the middle region of the crystals grown twice and three times.

As shown in Figure 4, the impurities concentration $C_{2}(x=$ 2) $\sim 110 \%$, in the middle of the crystal, is greater than that found in the raw material $\left(C_{2,0}=100 \%\right)$. The proposed model (Figure 1) provides the comprehension of this effect, since it is capable of forecasting the notion that the impurities located in the prior region migrate to the subsequent region, contributing to the increase of their concentration. Thus, the $k_{i, j}$ parameter is valuable to represent the effectiveness of the purification technique. Moreover, the model may be useful to predict the repetition number of Bridgman growths required to reduce the impurities to a level, for example, of $10 \%$ of the raw salt $\left(C_{0}=100 \%\right)$. In the present work, approximately 10 repetitions of the Bridgman growth would be necessary (Figure 5) to achieve this requirement.

For the purpose of analyzing the effectiveness of the purification process, a spectrometric analysis was performed 


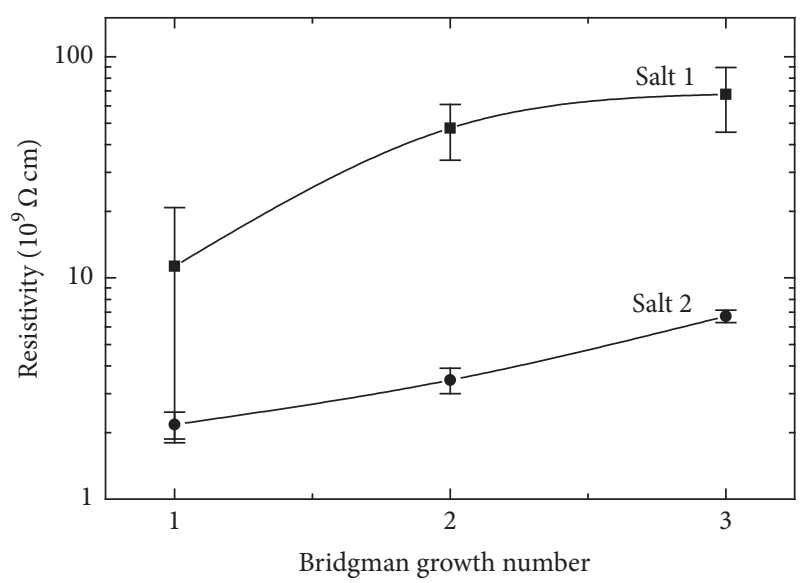

FIGURE 7: Resistivity of the $\mathrm{TlBr}$ detector from crystal middle region (slice $\sim 0.65 \mathrm{~mm}$ thick) as a function of the repetition number of Bridgman growth. The bar error represents one standard deviation ( $N=3$ samples).

TABLE 3: Resistivity values for $\mathrm{TlBr}$ detectors prepared from the crystal grown once, twice, and three times by the repeated Bridgman method. The samples used are from crystal middle region.

\begin{tabular}{lcc}
\hline \multirow{2}{*}{ Bridgman growth } & \multicolumn{2}{c}{ Resistivity $\left(10^{9} \Omega \mathrm{cm}\right)$} \\
& Salt 1 & Salt 2 \\
\hline First & $11.3 \pm 9.5$ & $2.17 \pm 0.30$ \\
Second & $47.5 \pm 13.4$ & $3.45 \pm 0.45$ \\
Third & $67.5 \pm 21.9$ & $6.72 \pm 0.43$ \\
\hline
\end{tabular}

and the results were compared with the efficiency of the purification (Figure 6). The pulse height spectra obtained suggest a significant improvement in their profiles when the purification number is increased. For the TlBr crystal grown once, it was not possible to observe the photopeak profile because the pulses generated fall in the electrical noise region. For the $\mathrm{TlBr}$ grown twice, only the photopeak of $59 \mathrm{keV}$ of ${ }^{241} \mathrm{Am}$ gamma source can be observed. For the third grown crystal, some ranges of energy below $59 \mathrm{keV}$ can be observed. Both starting materials (Salts 1 and 2) show similar spectrum details, although the raw material of Salt 1 shows, systematically, better results, mainly in terms of resistivity values (Table 3 and Figure 7). The resistivity found in this work is similar to that described by Hitomi et al. [6].

The resistivity curve showed a positive slope (Figure 7), tending to achieve a plateau. Although both salts had, nominally, the same initial purity (99.99\%), the crystals grown showed resistivity differences of, approximately, 10 times. The resistivity of the crystals from Salt 1 presented values 10 times higher than crystals from Salt 2 (Figure 7 and Table 1). There is evidence that Salt 1 has better performance in all parameters studied: this fact could be associated with the resistivity of crystals. In fact, the lowest resistivity of the crystals produced with Salt 2 could be correlated with its lower performance. However, comparing the spectrometric performance, while the crystals from Salt 1 first growth (high resistivity) did not show any detailed spectrum, in contrast, Salt 2 second and third growths (low resistivities) presented good detailed spectra (Figure 6). This fact suggests that the resistivity did not seem to have a fundamental role in characterizing the spectrum quality of the crystal. Vieira et al. [7]. described a similar observation in their result of resistivity measurements correlated to the number of zone-refining passes carried out in the $\mathrm{TlBr}$ purification. Further studies should be carried out to elucidate these results.

\section{Conclusion}

The repeated Bridgman method was efficient to purify the $\mathrm{TlBr}$ crystals and to improve their performance as radiation detectors. A compartmental model defined by linear differential equations may be used to calculate the coefficients for the migration of impurities. This is useful for predicting the number of repetitions by Bridgman growth needed to achieve a desirable concentration value. The resistivity showed a positive slope, tending to reach a plateau after the third growth. The TlBr resistivity above $\sim 3 \mathrm{M} \Omega$ was almost unaffected by the number of repetitions when growing crystals by Bridgman technique.

\section{Competing Interests}

The authors declare that they have no competing interests.

\section{Acknowledgments}

The authors are grateful to $\mathrm{CNPq}$ for the financial support and grants.

\section{References}

[1] D. S. Macgregor and H. Hermon, "Room-temperature compound semiconductor radiation detectors," Nuclear Instruments and Methods in Physics Research Section A, vol. 395, no. 1, pp. 101-124, 1997.

[2] I. B. Oliveira, F. E. Costa, J. F. D. Chubaci, and M. M. Hamada, "Purification and preparation of $\mathrm{TlBr}$ crystals for room temperature radiation detector applications," IEEE Transactions on Nuclear Science, vol. 51, no. 3, pp. 1224-1228, 2004.

[3] K. Hitomi, M. Matsumoto, O. Muroi, T. Shoji, and Y. Hiratate, "Characterization of thallium bromide crystals for radiation detector applications," Journal of Crystal Growth, vol. 225, no. 2-4, pp. 129-133, 2001.

[4] V. Kozlov, H. Andersson, V. Gostilo et al., "Improvements and problems of Bridgman-Stockbarger method for fabrication of TlBr single crystal detectors," Nuclear Instruments and Methods in Physics Research A, vol. 607, no. 1, pp. 126-128, 2009.

[5] M. S. Kouznetsov, I. S. Lisitsky, S. I. Zatoloka, and V. V. Gostilo, "Development of the technology for growing $\mathrm{TlBr}$ detector crystals," Nuclear Instruments and Methods in Physics Research Section A: Accelerators, Spectrometers, Detectors and Associated Equipment, vol. 531, no. 1-2, pp. 174-180, 2004.

[6] K. Hitomi, T. Onodera, and T. Shoji, "Influence of zone purification process on $\mathrm{TlBr}$ crystals for radiation detector fabrication," Nuclear Instruments and Methods in Physics Research Section A: Accelerators, Spectrometers, Detectors and Associated Equipment, vol. 579, no. 1, pp. 153-156, 2007. 
[7] C. L. Vieira, F. E. Costa, and M. M. Hamada, "Effect of etching on the TlBr crystal surface and its radiation response," in Proceedings of the International Nuclear Atlantic Conference, VIII ENAN Proceedings, Santos, Brazil, October 2007.

[8] J. Vaitkus, V. Gostilo, R. Jasinskaite et al., "Investigation of degradation of electrical and photoelectrical properties in $\mathrm{TlBr}$ crystals," Nuclear Instruments and Methods in Physics Research, Section A: Accelerators, Spectrometers, Detectors and Associated Equipment, vol. 531, no. 1-2, pp. 192-196, 2004.

[9] F. E. Da Costa, P. R. Rela, I. B. De Oliveira, M. C. C. Pereira, and M. M. Hamada, "Surgical gamma probe with $\mathrm{TlBr}$ semiconductor for identification of sentinel lymph node," IEEE Transactions on Nuclear Science, vol. 53, no. 3, pp. 1403-1407, 2006.

[10] V. Kozlov, M. Heikkilä, P. Kostamo, H. Lipsanen, and M. Leskelä, "TlBr purification and single crystal growth for the detector applications," Nuclear Instruments and Methods in Physics Research, Section A: Accelerators, Spectrometers, Detectors and Associated Equipment, vol. 633, no. 1, pp. S72-S74, 2011.

[11] T. Tada, K. Hitomi, T. Tanaka et al., "Digital pulse processing and electronic noise analysis for improving energy resolutions in planar TlBr detectors," Nuclear Instruments and Methods in Physics Research A, vol. 638, no. 1, pp. 92-95, 2011.

[12] A. Ray, H. M. Smith III, and N. M. Haegel, "Temperature dependence of the indirect bandgap in thallium bromide from cathodoluminescence spectroscopy," Journal of Applied Physics, vol. 115, no. 16, Article ID 163709, 2014.

[13] M. Abramowitz and I. A. Stegun, "Laplace transform," in Handbook of Mathematical Functions with Formulas. Graphs and Mathematical Tables, chapter 29, pp. 1019-1030, Dover, New York, NY, USA, 9th edition, 1972.

[14] M. Berman, "The formulation and testing of models," Annals of the New York Academy of Sciences, vol. 108, no. 1, pp. 182-194, 1963.

[15] A. Rescigno and G. Segre, Drug and Tracer Kinetics, Blaisdell Publishing, Waltham, Mass, USA, 1966.

[16] C. H. Mesquita, AnaComp-Compartmental Analysis Aided by Computer-User Manual, Instituto de Pesquisas Energéticas e Nucleares, São Paulo, Brazil, 1994.

[17] F. E. da Costa, C. H. de Mesquita, and M. M. Hamada, "Temperature dependence in the long-term stability of the $\mathrm{TlBr}$ detector," IEEE Transactions on Nuclear Science, vol. 56, no. 4, pp. 1817-1822, 2009.

[18] R. A. Santos, J. B. R. Silva, R. F. Gennari et al., "Multi-elemental segregation analysis of thallium bromide impurities purified by the repeated bridgman technique," IEEE Transactions on Nuclear Science, pp. 4118-4123, 2012.

[19] W. G. Pfann, Zone Melting, John Wiley \& Sons, New York, NY, USA, 1958.

[20] P. R. Bevington, Data Reduction and Error Analisys for the Physical Sciences, McGraw-Hill, New York, NY, USA, 1969. 

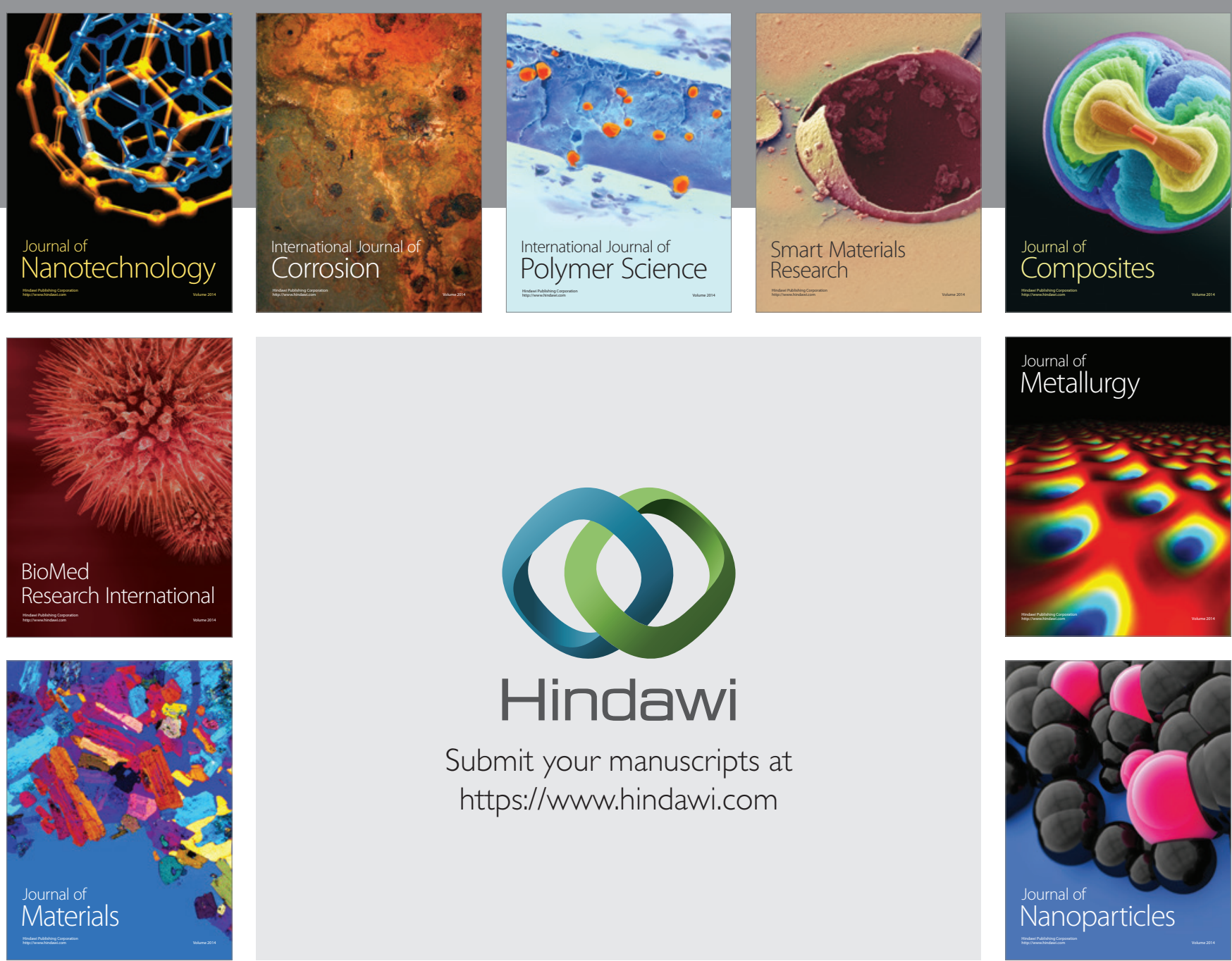

\section{Hindawi}

Submit your manuscripts at

https://www.hindawi.com

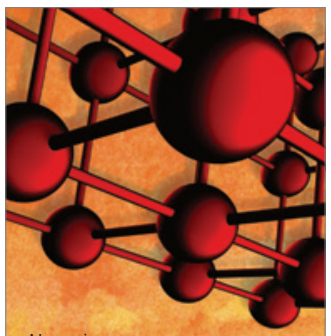

Materials Science and Engineering
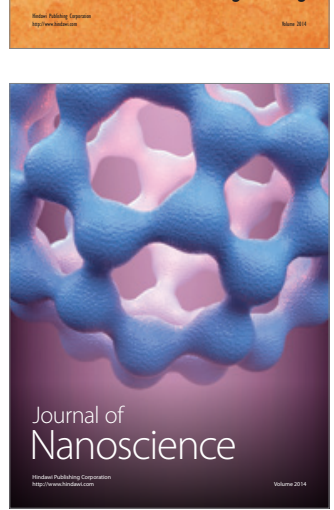
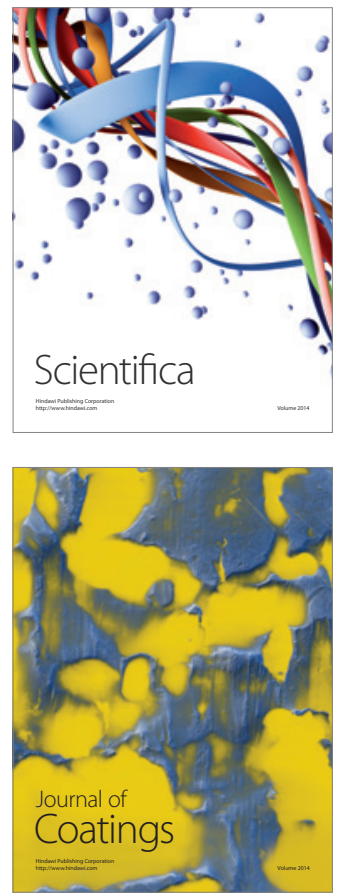
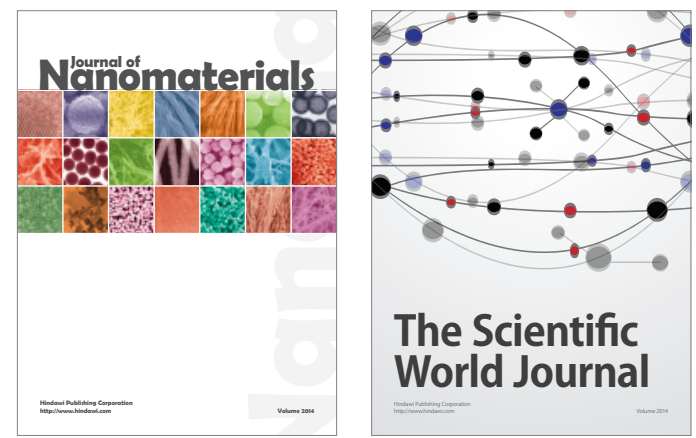

The Scientific World Journal
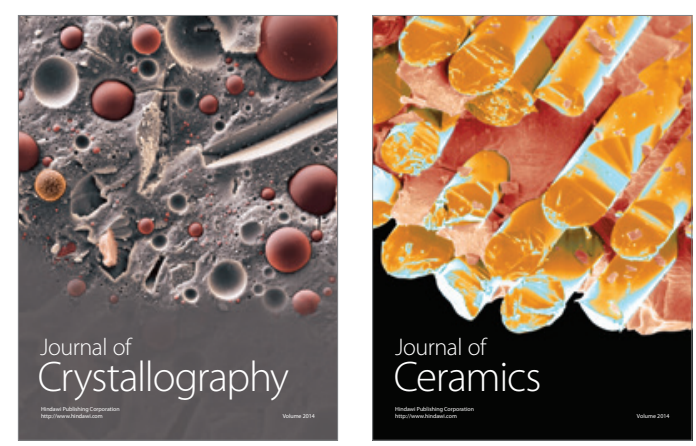
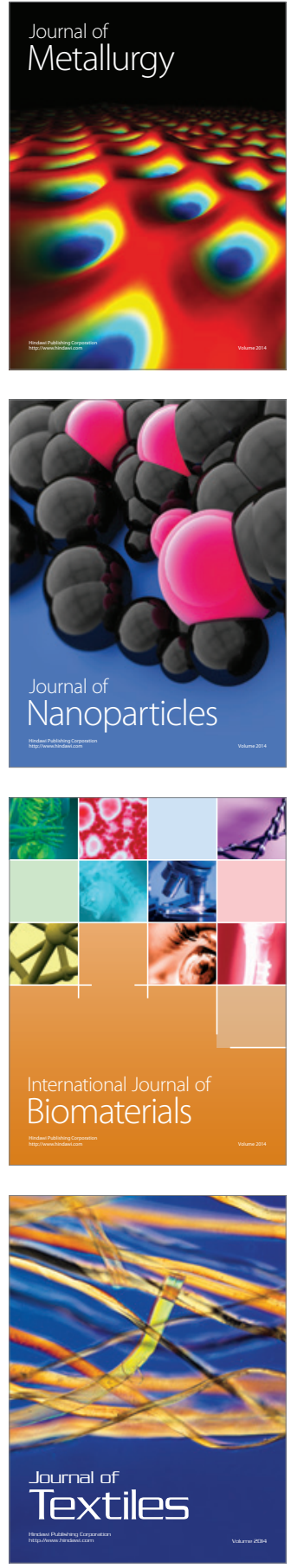